\title{
5-Aminolevulinic acid-induced protoporphyrin IX fluorescence as immediate intraoperative indicator to improve the safety of malignant or high-grade brain tumor diagnosis in frameless stereotactic biopsies
}

\author{
Gord von Campe • Michael Moschopulos • Martin Hefti
}

Received: 5 October 2011 / Accepted: 19 January 2012 / Published online: 2 February 2012

(C) Springer-Verlag 2012

\begin{abstract}
Background Frameless stereotactic biopsies are replacing frame-based stereotaxy as a diagnostic approach to brain lesions. In order to avoid a sampling bias or negative histology, multiple specimens are often taken. This in turn increases the risk of hemorrhagic complications.

Objective We present the use of 5-aminolevulinic acid (5ALA)-induced protoporphyrin IX fluorescence in frameless stereotaxy to improve the procedure duration and yield, and thereby reduce the risk of complications.

Methods Patients with suspected high-grade brain tumors are given 5-ALA $4 \mathrm{~h}$ prior to stereotactic biopsy. The biopsy needle is guided to the target using frameless stereotaxy based either on preoperative images or combined with intraoperative MRI sequences. The specimen is illuminated with blue light to look for fluorescence. In case of a positive fluorescence within the tissue sample, no frozen sections are obtained, and no further specimens are taken.

Results The samples of 13 patients revealed a positive fluorescence and were histologically confirmed as malignant or high-grade brain neoplasms. four cases were fluorescence-negative, requiring frozen section confirmation
\end{abstract}

\author{
G. von Campe \\ Universitätsklinik für Neurochirurgie, \\ Medizinische Universität Graz, \\ Graz, Austria \\ M. Moschopulos \\ Department of Neuropathology, Kantonsspital Aarau, \\ Aarau, Switzerland \\ M. Hefti $(\square)$ \\ Neurochirurgisches Zentrum, Klinik Am Rosenberg, \\ Hasenbühlstrasse 11, \\ 9410 Heiden, Switzerland \\ e-mail: martin.hefti@hirslanden.ch
}

and/or multiple samples. In theses cases histology was either nonspecific gliotic changes or low-grade tumors. There were no complications related to the additional use of 5-ALA.

Conclusion 5-ALA fluorescence in stereotactic biopsies can increase the safety and accuracy of these procedures by reducing sampling errors and eliminating the need for multiple samples and/or frozen section verification, creating a more accurate, faster and safer procedure for cases of suspected malignant or high-grade brain tumors situated in deep or eloquent areas.

Keywords 5-Aminolevulinic acid - Protoporphyrin IX · Stereotactic biopsy $\cdot$ Brain tumor $\cdot$ Fluorescence

\section{Introduction}

Over the last few years, frameless stereotactic biopsies have progressively replaced frame-based stereotactic procedures as a first line diagnostic approach to various brain lesions. The combined use of intraoperative magnetic resonance imaging (MRI) further increases the spatial resolution of the probe target correlation in these procedures [7]. Yet, despite the increasing accuracy of frameless stereotactic procedures, failure to correctly establish tumor histology and dignity occurs in approximately $25 \%$ of cases, mainly due to tissue heterogeneity and sampling bias [3, 7]. In cases where the frameless stereotactic biopsy is based solely on preoperative computertomographic (CT) or MRI images, the results are even less favorable with $37 \%$ [1] to $49 \%$ [5] of incorrectly diagnosed gliomas. In order to avoid sampling bias, obtaining multiple specimens has been advocated [12]. Since stereotactic procedures are the diagnostic method of choice for deep-seated lesions, within the brain stem, or 
within the so-called eloquent areas, taking additional samples during the same procedure to improve the diagnostic accuracy can be risky or even impossible. A precise histological diagnosis, however, is crucial for appropriate treatment guidance and prognosis. To decrease the morbidity and mortality risks associated with multiple sampling, we introduced fluorescence-controlled stereotaxy in our department [4]; additional cases were published in support of the concept by others [8]. We here present a series of 17 consecutive patients where we combined 5-aminolevulinic acid (5-ALA)-induced fluorescence with frameless stereotaxy for rapid biopsy accuracy verification in cases of suspected malignant or high-grade brain tumors.

\section{Methods}

Four hours prior to surgery the patient is given an oral dose of a $20-\mathrm{mg} / \mathrm{kg}$ body weight aqueous solution of 5 aminolevulinic acid hydrochloride (Gliolan ${ }^{\circledR}$ medac $\mathrm{GmbH}$, Hamburg, Germany) [11]. The MRI data used for biopsy planning and guidance are obtained either preoperatively using a standardized high-resolution imaging protocol or, if available, intraoperatively after the patient has been positioned on the operating table. After general anesthesia, burr hole placement and dural opening at the determined entry point are performed in the usual fashion. Using frameless stereotaxy navigation (StealthStation ${ }^{\circledR}$, Medtronic, Louisville, KY, USA), the biopsy needle is then advanced into the targeted lesion, guided by the MRI data, and a first sample is taken. After removal of the needle it is placed under a 405-440-nm blue light source (either a dedicated light source or the light source of an operating microscope fitted for 5-ALA-induced PpIX fluorescence) with the specimen still in place to look for fluorescence. In case of strong positive fluorescence, no frozen section is obtained, and no further samples are taken.

\section{Illustrative case}

A 71-year-old male (Table 1, case 01) with multiple intracerebral lesions of unknown origin underwent a frameless stereotactic biopsy in the periventricular area of the right frontal horn. As a proof of concept, the accuracy of the calculations and needle placement was verified by obtaining an intraoperative MRI scan with the titanium biopsy needle (Pajunk Medizintechnologie, Geisingen, Germany) still in place. After removal of the needle and blue light illumination of the tissue sample, a positive red fluorescence could be observed. As the opening of the biopsy needle $(10 \mathrm{~mm})$ was larger than the target lesion $(5 \mathrm{~mm})$, and the needle itself has a 2-mm forerun (Fig. 1, top), only the distal part of
Table 1 Demographic data, detected fluorescence and final pathology of 17 patients who underwent a frameless stereotactic brain biopsy after oral administration of Gliolan ${ }^{\circledR}$ (5-ALA). Fluorescence was assessed under a 440-nm blue light source

\begin{tabular}{|c|c|c|c|c|}
\hline Patient & Sex & $\begin{array}{l}\text { Age } \\
\text { (years) }\end{array}$ & Fluorescence & Final histology \\
\hline 01 & M & 71 & Positive & B-cell lymphoma \\
\hline 02 & M & 60 & Positive & $\begin{array}{c}\text { Glioblastoma } \\
\text { multiforme }\end{array}$ \\
\hline 03 & M & 79 & Positive & $\begin{array}{c}\text { Glioblastoma } \\
\text { multiforme }\end{array}$ \\
\hline 05 & M & 70 & Positive & $\begin{array}{c}\text { Glioblastoma } \\
\text { multiforme }\end{array}$ \\
\hline 07 & $\mathrm{~F}$ & 38 & Positive & B-cell lymphoma \\
\hline 09 & M & 25 & Positive & Germinoma \\
\hline 10 & M & 49 & Positive & $\begin{array}{l}\text { Astrocytoma WHO } \\
\text { grade III }\end{array}$ \\
\hline 11 & $\mathrm{~F}$ & 72 & Positive & $\begin{array}{l}\text { Astrocytoma WHO } \\
\text { grade III }\end{array}$ \\
\hline 12 & $\mathrm{~F}$ & 76 & Positive & B-cell lymphoma \\
\hline 13 & M & 65 & Positive & B-cell lymphoma \\
\hline 15 & M & 72 & Positive & $\begin{array}{c}\text { Glioblastoma } \\
\text { multiforme }\end{array}$ \\
\hline 16 & M & 65 & Positive & $\begin{array}{c}\text { Glioblastoma } \\
\text { multiforme }\end{array}$ \\
\hline 17 & $\mathrm{~F}$ & 39 & Positive & $\begin{array}{c}\text { Glioblastoma } \\
\text { multiforme }\end{array}$ \\
\hline 04 & M & 30 & Negative & $\begin{array}{l}\text { Astrocytoma WHO } \\
\text { grade II }\end{array}$ \\
\hline 06 & $\mathrm{~F}$ & 71 & Negative & $\begin{array}{l}\text { Astrocytoma WHO } \\
\text { grade II }\end{array}$ \\
\hline 08 & M & 52 & Negative & Unspecific gliosis \\
\hline 14 & M & 82 & Negative & Unspecific gliosis \\
\hline
\end{tabular}

Age in years, $M=$ male, $F=$ female.

the actual specimen was expected to hold pathological tissue, which was confirmed by visible 5-ALA-induced protoporphyrin IX (PpIX) fluorescence only within the tumor tissue (Fig. 1, bottom). Histology and immunohistochemistry revealed a malignant lymphoma (B-cell lympho$\mathrm{ma}$ ), and showed the border between the target lesion and the surrounding brain (Fig. 2) as predicted by fluorescence and MRI.

\section{Results}

Seventeen biopsies using a combination of frameless stereotaxy and 5-ALA-induced fluorescence were performed (Table 1). In 13 cases a positive fluorescence was detected and histological examination confirmed a malignant or high-grade brain neoplasm in all cases. The specimens of the remaining four biopsies were 


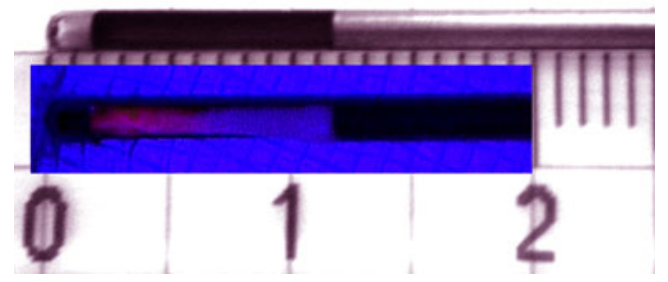

Fig. 1 The opening of the biopsy needle $(10 \mathrm{~mm})$ is larger than the target lesion $(5 \mathrm{~mm})$, and the needle itself has a 2-mm fore-run (Fig. 1, top). Only the distal part of the actual specimen was expected to hold pathological tissue, which was confirmed by visible 5-ALA-induced protoporphyrin IX (PpIX) fluorescence only within the tumor tissue (Fig. 1, bottom)

fluorescence-negative and required frozen section confirmation and/or further samples. In these cases histology showed either nonspecific gliotic changes or low-grade tumors, both of which are known not to induce any fluorescence after 5-ALA application.

In the fluorescence-positive cases the procedure time could be reduced by $30-45 \mathrm{~min}$, since no frozen sections needed to be obtained and no resampling was necessary.

In this small series of patients there were no complications related either to the use of 5-ALA or to the surgical procedure itself.

\section{Discussion}

Stereotactic brain biopsies carry a morbidity and mortality risk of $4 \%$ and $0.9 \%$, respectively, and lead to an inaccurate or imprecise diagnosis in one third to one half of glioma cases [5]. Further improvement can be achieved by obtaining multiple specimens and/or the additional use of intraoperative imaging [7]. Since patients with malignant gliomas fare better with gross total resection [6], bioptic procedures are usually limited to inoperable or small lesions that are deeply located (e.g., brain stem) or situated in eloquent

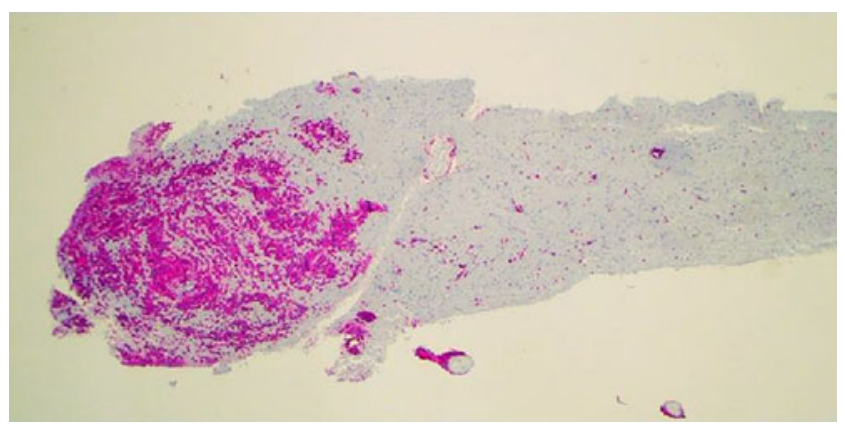

Fig. 2 Immunohistochemistry revealed a malignant lymphoma (B-cell lymphoma), and showed the border between the target lesion and the surrounding brain (CD 20) cortical areas. Unfortunately, these typical areas for biopsy are the least suitable for multiple tissue sampling. Intraoperative frozen sections can aid the neurosurgeon in verifying the adequate biopsy sampling location, but they are time consuming and also prone to errors.

5-ALA is a non-fluorescent "pro-drug" that, due to a metabolism disturbance, is transformed into fluorescent PpIX in the tumor cells of high-grade gliomas [10] and other highly proliferative neoplasms. This property is used to achieve radical tumor resection and thereby extend progression-free survival in high-grade glioma patients [11]. In the case of stereotactic brain biopsy, the use of protoporphyrin fluorescence does not aim at optimal resection, but allows the procedure to be time-efficient and as minimally invasive as possible by reducing the risk of multiple tumor samplings. The sensitivity and specificity of malignant tissue detection have been reported to be $85 \%$ and $100 \%$, respectively [2]. The PPV of observable intraoperative PpIX fluorescence for non-normal tissue is found to be as high as $98.9 \%$ [9]. Therefore, a sample showing strong positive fluorescence most likely represents the targeted lesion. Although we used intraoperative MR imaging (PoleStar N20 ${ }^{\circledR}$, Medtronic, Yokneam Elit, Israel) for navigation to assess the accuracy of the method described and to rule-out an acute hemorrhagic complication, the same methodology is applicable to frameless stereotactic procedures based on preoperative CT or MR images, the main purpose being that no further samplings are required in case of a positive fluorescence.

Negative fluorescence can either be due to a sampling error (small lesion, preoperative imaging not accounting for brain shift) or to the histological nature of the pathology (non-fluorescent tumor or lesion). In these cases frozen sections are required to assess the adequacy of the sampling location; if available, intraoperative imaging can also be used to correct the needle position relative to the intended target and can be of invaluable help in very small lesions.

\section{Conclusion}

The addition of 5-ALA-induced PpIX fluorescence proved useful in frameless stereotactic biopsies of malignant or high-grade brain tumors where immediate strong intraoperative fluorescence was observed. The procedure time could be reduced and its safety increased by eliminating the need for multiple samples. In these cases frozen section verification may not be mandatory, since the final pathology was conclusive in all instances of positive fluorescence. The method is easy to implement and requires no modification to the biopsy procedure itself. Fluorescencenegative cases represent either a missed target or a nonfluorescent pathology, and therefore do require frozen sections 
and/or intraoperative imaging to verify the adequacy of the sampled target.

\section{Conflicts of interest None.}

\section{References}

1. Chandrasoma PT, Smith MM, Apuzzo ML (1989) Stereotactic biopsy in the diagnosis of brain masses: Comparison of results of biopsy and resected specimen. Neurosurgery 17:12-18

2. Friesen SA, Hjortland GO, Madsen SJ, Hirschberg H, Engebraten O, Nesland JM, Peng Q (2002) 5-Aminolevulinic acid based photodynamic detection and therapy of brain tumors (Review). Int J Oncol 21:577-582

3. Glantz MJ, Burger PC, Herndon JE II, Friedman AH, Cairncross JG, Vick NA, Schold JR (1991) Influence of the type of surgery on the histologic diagnosis in patients with anaplastic gliomas. Neurology 41:1741-1744

4. Hefti M, von Campe G, Moschopulos M, Siegner A, Looser H, Landolt H (2008) 5-aminolevulinic acid induced protoporphyrin IX fluorescence in high-grade glioma surgery: a one-year experience at a single institution. Swiss Med Wkly 138(11-12):180-185

5. Jackson RJ, Fuller GN, Abi-Said D, Lang FF, Gokaslan ZL, Shi WM, Wildrick DM, Sawaya R (2001) Limitations of stereotactic biopsy in the initial management of gliomas. Neuro Oncol: 193-200

6. Lacroix M, Abi-Said D, Fourney D, GokaslanZL SW, DeMonte F, Lang FF, McCutcheon IE, Hassenbusch SJ, Holland E, Hess K, Michael C, Miller S, Sawaya R (2001) A multivariate analysis of 416 patients with glioblastoma multiforme. Prognosis, extend of resection and survival. J Neurosurg 95:190-198

7. Moriarty TM, Quinones-Hinojosa A, Larson PS, Alexander E 3rd, Gleason PL, Schwartz RB, Jolesz FA, Black PM (2000) Frameless stereotactic neurosurgery using intraoperative magnetic resonance imaging: stereotactic brain biopsy. Neurosurgery 47(5):1145-1146

8. Moriuchi S, Yamanda K, Dehara M, Teramoto Y, Soda T, Imakita M, Taneda M (2011) Use of 5-aminolevulinic acid for the confirmation of deep-seated brain tumrs during atereotactic biopsy. Report of 2 cases. J Neurosurg 115(2):278-280

9. Roberts DW, Valdes PA, Harris BT, Fontaine KM, Hartov A, Fan X, Ji S, Lollis SS, Pogue BW, Leblond F, Tosteson TD, Wilson
BC, Paulsen KD (2011) Coregistered fluorescence-enhanced tumor resection of malignant glioma: relationships between deltaaminolevulinic acid-induced protoporphyrin IX fluorescence, magnetic resonance imaging enhancement, and neuropathological parameters. Clinical article. J Neurosurg 114(3):595-603

10. Stummer W, Stepp H, Möller G, Erhardt A, Leonhard M, Reulen HJ (1998) Technical principles for protoporphyrin-IX-fluorescence guided microsurgical resection of malignant glioma tissue. Acta Neurochir 140:995-1000

11. Stummer W, Pichlmeier U, Meinel Th, Wiestler OD, Zanella F, Reulen HJ (2006) Fluorescence-guided surgery with 5aminolevulinic acid for resection of malignant glioma: A randomised controlled multicenter phase III trial. Lancet Oncol 07:392401

12. Woodworth G, Mc Girt MJ, Samdani A, Garonzik I, Olivi A, Weingart JD (2005) Accuracy of frameless and frame-based guided stereotactic brain biopsy in the diagnosis of glioma: Comparison of biopsy and open resection specimen. Neurol Res 27(4):358 362

\section{Comment}

The authors obtained frameless stereotactic needle biopsies from brain lesions of 17 patients $4 \mathrm{~h}$ after 5-ALA administration. In the operating room, biopsy samples still in the needle were placed under blue light $(405-440 \mathrm{~nm})$, which is generally available on operation microscopes fitted for the 5-ALA method. Samples of 13 tumors became pink, and all 13 were histologically malignant. The practical point is - taken that pink almost always indicates a malignant tumor - that no further samples are needed after a pink one. The authors saved 30 to $45 \mathrm{~min}$ of procedure time, not needing to wait for frozen section diagnoses and not taking more samples. This is a clever diagnostic extension of the 5ALA method.

In the next wave of intraoperative diagnostics with biologically targeted fluorescent molecules, we should have light sources in the operation microscopes that provide illumination and excitation at desired and changeable wave length bands, and we should have spectral analysis tools to better distinguish diagnostic fluorescence among the light that reflects/scatters from the tissues in the operative field.

Juha E. Jääskeläinen

Kuopio, Finland 